score was associated with both internalising $(B=0.22,95 \% \mathrm{CI}$ $\left.0.08-0.36, \quad \mathrm{p}<0.01, \mathrm{f}^{2}=0.03\right)$ and externalising symptoms $\left(\mathrm{B}=0.40,95 \%\right.$ CI $\left.0.20-0.61, \mathrm{p}<0.001, \mathrm{f}^{2}=0.05\right)$, but not with cognitive outcomes in toddlers $(B=-0.21$, 95\% CI $-0.49-$ $0.06, \mathrm{p}>0.05)$. Maternal EPDS, and toddlers' CBCL and QCHAT scores did not differ between preterm and term participants.

Conclusions Our findings indicate that children whose mothers had increased depressive symptoms in the early postnatal period exhibit more maternally-reported behavioural, emotional and neurodevelopmental problems in toddlerhood. Further research is needed to confirm the clinical significance of these findings.

\section{CONTINUOUS INFUSION OF VANCOMYCIN IMPROVED THERAPEUTIC LEVELS IN TERM AND PRETERM INFANTS}

Antonia Round, Khuen Foong Ng. UK

\subsection{6/bmjpo-2021-RCPCH.79}

Background Growing evidence suggests that continuous infusion of vancomycin (CIV) is superior to intermittent infusion of vancomycin (IIV) in neonates. Prescribing CIV in neonates in the UK remains relatively untested and is an unlicensed use. This quality improvement (QI) project aimed to transition from IIV to CIV with earlier and improved attainment of therapeutic vancomycin levels.

Objectives This project aimed to clarify the utility, benefit and safety of CIV over current IIV usage in our department, aiming to inform future vancomycin regimens both locally and more widely. Following baseline measurements of IIV, a QI project was undertaken for implementation of CIV using sequential Plan Do Study Act (PDSA) cycles over 10 months (July 2019 to May 2020) with Specific, Measurable, Achievable, Realistic and Timely (SMART) aims to:

1. Reduce the time taken to achieve vancomycin target level by $50 \%$.

2. To increase first TDM levels within target range from $36 \%$ to $60 \%$.

3. To increase total TDM therapeutic levels from $44 \%$ to $70 \%$ in neonates.

If successful, this would add to current evidence that CIV is superior to IIV in neonates and should be regarded as best practice.

Methods The Model for Improvement framework with Plan Do Study Act cycles was used. Prospective data were collected during three phases: IIV, CIV-1 and CIV-2.

Results Using IIV, 36\% (9/25) of first vancomycin levels were within target range. CIV achieved therapeutic levels twice as quickly as IIV $(\mathrm{p}<0.05)$ with improved first vancomycin target levels (IIV 36\%, 9/25; CIV-1 55\%, 16/29; CIV-2 61\%, $14 / 23$ ) and total therapeutic levels (IIV 44\%, 37/84; CIV-1 56\%, 55/98; CIV-2 69\%, 79/114).

Conclusions It was established that IIV outcomes were not meeting expected standards, particularly in terms of delayed time to therapeutic levels and high percentage of out of range levels. This project was successful in establishing a change to CIV which produced a modest benefit over IIV, particularly in regard to time taken to achieve vancomycin target levels and proportion of therapeutic levels. The findings of this project and information regarding our implementation process provide guidance to other NICUs to transition from IIV to CIV.

\section{HOSPITAL MORTALITY IN PATIENTS WITH RARE DISEASES DURING THE COVID-19 AND SARS PANDEMICS: RESULTS FROM A 7.5 MILLION POPULATION}

Claudia Ching Yan Chung, Wilfred Hing Sang Wong, Brian Hon Yin Chung. Hong Kong

\subsection{6/bmipo-2021-RCPCH.80}

Background The threat of pandemics occur differently for different groups. The rare disease population is at particular risk of being further marginalised during pandemics.

Objectives To assess hospital mortality in the rare disease and general populations during the coronavirus disease of 2019 (COVID-19) and severe acute respiratory syndrome (SARS) pandemics in the 7.5 million population in Hong Kong.

Methods Using the Clinical Data Analysis and Reporting System (CDARS), a population-level database that records all public healthcare records in Hong Kong, all admission records during the COVID-19 (January 23 - August 23, 2020) and SARS (March 11 - June 30, 2003) pandemics were extracted. Patients with rare diseases were identified using one or more of the 1,084 10th version International Classification of Diseases and Related Health Problems (ICD10) codes cross-referenced with 467 ORPHAcodes. Admission records during the same period in 2019 and 2002 were retrieved for comparison. Primary outcomes were COVID-19/ SARS mortality in hospital. Secondary outcomes were overall hospital mortality during the COVID-19 and SARS pandemic periods. Subgroup analysis by age-group $(\leq 18,>18$ to $<60$, $\geq 60$ ) was performed to understand the mortality patterns. Logistic regression was used to estimate the odds ratios with 95\% confidence intervals (CIs).

Results During the COVID-19 pandemic, 407,219 patients were admitted to one or more of the 43 public hospitals in Hong Kong, of which, 39,576 (9.7\%) were paediatric patients $\leq 18$ years old, and $13,894(3.4 \%)$ were rare disease patients. Of the 4,381 patients admitted with COVID-19, $81(1.8 \%)$ died during the same admission, of which, 5 (6.2\%) were patients with rare diseases. COVID-19-related mortality was almost exclusively seen in patients $\geq 60$ years in both rare disease and general populations, with mortality being $21.7 \%$ and $7.4 \%$, respectively. None of the COVID19 patients $\leq 18$ years died by the time of data extraction. Patients with rare diseases had an adjusted 3.4 times odds of COVID-19-related hospital mortality compared with that of the general population (95\% CI 1.24-9.41). In contrast, 158,930 patients were admitted during the SARS pandemic, with $24,045(15.1 \%)$ being $\leq 18$ years and 5,249 (3.3\%) being rare disease patients. Of the 1,449 patients admitted with SARS, $234(16.1 \%)$ died during the same admission, of which, $1(0.4 \%)$ was $\leq 18$ years, and $6(2.6 \%)$ were patients with rare diseases. While age-related increase in mortality was observed for the general population during the SARS pandemic, this pattern was not observed in the rare disease population. Rare disease patients $\leq 18$ years had a 12.5 times higher SARS-related mortality than those in the general population $(12.5 \%$ vs $1.0 \%)$. Patients admitted during the same pandemic periods without coronavirus infection had a significantly higher hospital mortality 
compared with those admitted one year before the pandemics $(\mathrm{p}<0.001)$.

Conclusions This population-based study demonstrated the differential impacts of COVID-19 and SARS on rare disease patients in Hong Kong, a group that is currently not strategically protected. Real-time data analysis by age group within different populations could be of consideration when developing prioritisation guidelines in the future. Taken together, this study warrants cautious healthcare planning, with consideration of prioritising patients with rare diseases.

\section{TAKING THINKING TOGETHER FORWARD: A SCHEME LINKING PAEDIATRIC AND CAMHS TRAINEES}

Jo Cryer, Megan Fisher. UK

\subsection{6/bmjpo-2021-RCPCH.81}

Background The concept of joining up services and managing young people's mental health difficulties has never been more paramount. Covid has thrown us all into a world of uncertainty and this is exacerbated for young people with anxiety, underlying medical or psychiatric needs. At a time when linking people up to work and think together is the most needed, of course it is also the most challenging. Mental health presentations are an increasing part of the paediatric caseload and there is growing evidence that paediatric trainees feel ill-equipped to manage these patients. Equally CAMHS trainees reported they required greater access to seeing children under 5, assessing development and physical health. Only $33 \%$ of trainees we surveyed felt that their current training programme enabled them to achieve their curriculum requirements in paediatrics or mental health respectively.

Objectives We developed the concept of Thinking Together, to tackle this training gap. The scheme involves pairing paediatric and CAMHS trainees to share clinical encounters to foster a joint way of learning and working together, coming together to look at how we can link training to optimise patient care. CAMHS services are increasingly stretched, raising referral thresholds and making the need for closer working together across disciplines paramount.

Methods A pilot was launched in March 2016 where 30 trainees from CAMHS and Paediatrics were paired for a period of 6 months, attending at least two clinical encounters in each setting. Trainees were encouraged to explore a variety of learning possibilities, including clinics, referral meetings and signposting their partner to other relevant clinical opportunities.

In 2018 we extended the scheme in a second phase pilot to four centres across the UK. Feedback from participants involved was equally positive but there were logistical challenges. We reflected on feedback from the initial and second phase pilot and adapted the programme in 2019/2020. We aimed to consolidate the scheme in one centre and include workshops, which allowed focus on a topic relevant to both specialties, in addition to a forum for debrief discussions on challenging cases.

Results Prior to participating in Thinking Together, 70\% stated they had no experience of working in a jointly delivered paediatric/mental health clinic. $93 \%$ of participants felt that their patients benefitted from access to jointly delivered clinics. Feedback was very positive and identified that the scheme met training gaps for both specialties. Th adapted version of Thinking Together that we rolled out in 20192020 was also met with positive feedback and proved to be particularly beneficial during the pandemic, when trainee pairs could still participate in Thinking Together via virtual workshops.

Conclusions Covid has clearly brought challenges to every aspect of our lives but this is a training model that has been able to adapt in this unprecedented time, by means of virtual workshops and the enthusiasm of the participants to continue to pursue learning opportunities, including joining referral meetings remotely. Thinking Together has evolved into a successful model that we would like to embed in everyday practice.

\section{REDUCING NEONATAL INTENSIVE CARE ADMISSIONS OF TERM INFANTS DURING COVID-19}

Sina Durani, Lesley Alsford. UK

\subsection{6/bmjpo-2021-RCPCH.82}

Background Covid-19 is a novel disease which has had an impact on the delivery of health care services, including postnatal care. Term newborns may often require additional clinical input varying from postnatal wards to the neonatal intensive care unit.

Objectives To review the admissions of term neonates to a level 2 neonatal intensive care unit (NICU) and transitional care (TC) ward (a postnatal ward) during the COVID-19 period.

Methods A retrospective audit was carried out of term neonatal admissions to NICU and TC during 1st March 2019-31st August 2019 (Period 1) and compared with 1st March 2020 $31^{\text {st }}$ August 2020 (period 2; Covid - 19 period). Babies born at term $(>37 / 40)$ were included. Babies who were premature $(<37 / 40)$ or repatriated from other hospitals were excluded. This also included reviewing term babies re-admitted from home within 7 days of birth.

Results Admissions to NICU had reduced by $1 / 3$ (143 during period 1, 97 during period 2). Similarly, there was a marked reduction in admissions related to respiratory distress (64 in period 1, 42 in period 2) and infection (14 in period 1, 4 in period 2). There were only 2 direct admissions due to Covid19. There were similar numbers of admissions from home (31 in period 1, 35 in period 2). There was an increase in the number of babies admitted from D4-5 of life, yet NICU admissions from home had nearly halved (21 in Period 1, 11 in period 2). There was an increase in admissions to the TC ward instead (10 in Period 1, 24 in Period 2), including more babies requiring treatment for jaundice. The admissions to the TC ward were mostly not in line with the existing 'readmission from home policy' however there were no complications from these admissions. There was a reduction in the readmissions from home due to hypernatremic dehydration and infection.

Conclusions Although overall delivery numbers were similar between the two time periods, Elective C-sections occurred off site which may explain the reduction in admissions for respiratory distress. The improvement in isolation and infection protocols could explain the reduction in admissions due 